\title{
THE DIABETIC FOOT
}

\author{
R S Elkeles, John H N Wolfe
}

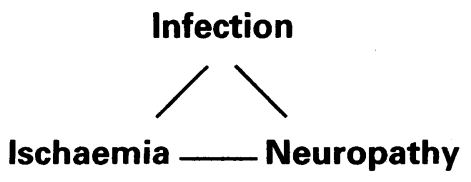

\section{The neuropathic foot}
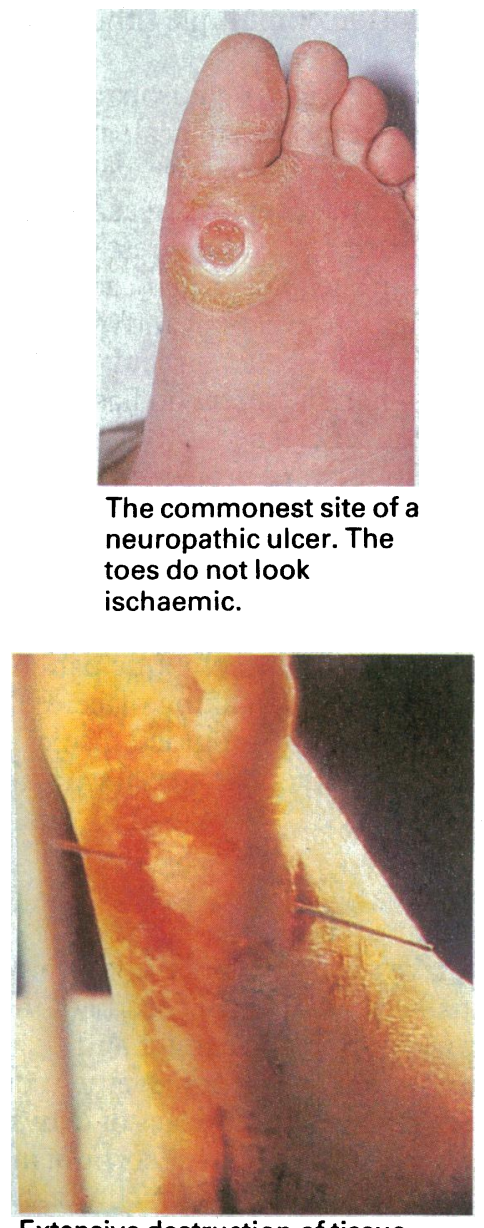

Extensive destruction of tissue beneath small lesions, with needle running along track between plantar and dorsal ulcers.
More hospital beds in Britain are occupied by diabetic patients with disorders of their feet than by patients with all the other complications of diabetes combined (despite the fact that diabetes is also one of the most common causes of both renal failure and blindness). The two main features are neuropathy and ischaemia, both of which predispose to infection and lead to necrosis of the tissue. They are often present together.

The management of the diabetic foot requires the combined skills of a number of people: general practitioner, diabetic physician, vascular surgeon, orthopaedic surgeon, chiropodist, nurse, microbiologist, physiotherapist, surgical footwear specialist, and limb fitter. Every effort should be made to make sure that this team works together.

The most common serious complication of diabetic peripheral neuropathy that affects the foot is the neuropathic ulcer. Loss of sensation results in failure to perceive damage caused by mechanical trauma - for example, friction from badly fitting shoes, penetration of sharp objects on the floor, or heat from radiators or fires. Neuropathy also stops the intrinsic muscles of the foot from opposing the long muscles, which leads to the "claw toe" deformity and transfers weight bearing from the toes to the heads of the metatarsals. Pressure on the plantar skin is increased and callus builds up under the heads of one or more metatarsals. Fluid collects underneath the callus and infection may then supervene, leading to abscess formation and ulceration. It is important to appreciate that - unlike with most other ulcers - the initial problem is the formation of a cavity deep to the epithelium, which is followed by ulceration. The initial event is the formation of a plaque of hard keratin, and the tissues break down underneath this. A cavity filled with plasma and blood then develops, enlarges, and eventually ruptures on to the skin surface. The ulcerated opening is therefore small compared with the large cavity beneath. This ulcer may remain uninfected for a prolonged period, but if the opening to the cavity becomes occluded with keratin and inspissated discharge, then overt infection may develop rapidly and progress at an alarming rate to affect the underlying tendon and bone.

The problem is further complicated by alteration to both the structure and function of proteins in diabetic patients. Hyperglycaemia produces non-enzymatic glycosylation of collagen and keratin. These tissues then become more rigid and inflexible, and resistant to enzymatic digestion by collagenase. Keratin is not readily removed from the superficial layers of the sole of the foot and hyperkeratosis develops. The abnormal collagen is highly inflexible so that shear stresses on the pressure points. lead to tissue breakdown and eventual ulceration.

Healing of this ulceration is also impaired in diabetic patients. Their raised tissue glucose concentrations reduce the activity of leucocytes and macrophages, which fail to produce enough fibroblasts to synthesise the collagen.

On clinical examination the diabetic neuropathic foot is warm, pink, and dry with easily palpable pulses. Tendon reflexes are impaired and sensation to vibration, pinprick, and light touch is reduced. 


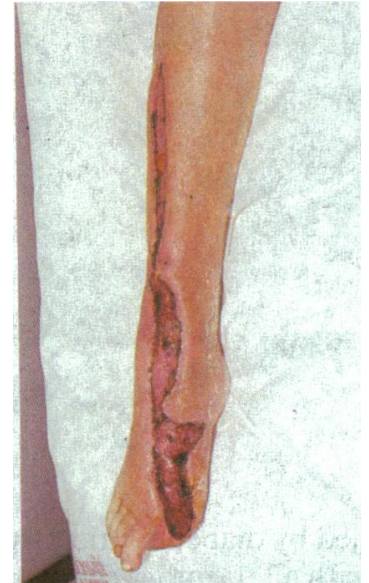

Extensive infection tracking up the anterior compartment of the leg. After debridement and skin grafting the leg was saved.

\section{Management}

Infection - Infections are almost invariably associated with a mixture of Gram positive, Gram negative, enteric, and anaerobic organisms. As about $70 \%$ of infected ulcers contain anaerobic organisms systemic treatment is essential. Swabs should be taken from the ulcer before antimicrobial agents are prescribed. If there is spreading cellulitis blood should be cultured. A patient with superficial ulceration can be treated with systemic antibiotics, cleansing of the ulcer, and application of non-adhesive dressings as an outpatient, but the importance of restricting activity until the inflammation has settled must be emphasised. More serious infections require admission to hospital for bed rest and antibiotics given intravenously. A combination of benzylpenicillin, ampicillin, and metronidazole is usually given until results of the cultures are available, when a more appropriate choice can be made.

Removal of necrotic tissue - A grossly infected neuropathic foot with osteomyelitis of the metatarsals can usually be saved, so any foot that is not ischaemic should be managed conservatively. Initially the infection should be drained and irrigated, frankly necrotic tissue should be removed, antibiotics given, and the patient confined to bed. An attempt to remove all the infected and inflamed tissue at this stage may result in unnecessary extensive destruction of tendons in the foot, and the resulting deformity may make it difficult to prevent further damage to pressure points. Once the infection has been drained and controlled it is possible to assess the extent of damage to the tissues, and the cavity can be carefully pared back to healthy tissue. Every effort should be made to preserve viable skin to close the defect.

Control of diabetes - It is likely that control of the diabetes helps to reduce the spread of infection, but control of diabetes is itself made more difficult by infection. A patient who has been treated with diet only or with diet and insulin tablets will usually require insulin during these periods of infection, given either by intravenous infusion or multiple subcutaneous injections.

Redistribution of weight-Neuropathic ulcers often take weeks or months to heal, and the only way to ensure healing is to remove weight bearing and friction from the ulcerated areas. In practice this is difficult. Bed rest is the surest way in the short term, but in the long term it can be extremely trying for the patient and (in these days of extreme shortage of beds) difficult and costly. An alternative when the infection has been treated and the ulcer cleaned is to apply a below knee plaster with forward projection of the cast to the level of the mid-shaft of the proximal phalanges of the toes. This permits the ulcer to be inspected and dressed. Because of the sensory neuropathy, extra foam padding must be put under the plaster. By this technique patients can be mobile while healing is taking place. Other methods include wearing moulded insoles or a semirigid boot of synthetic material with a piece cut out over the ulcer.

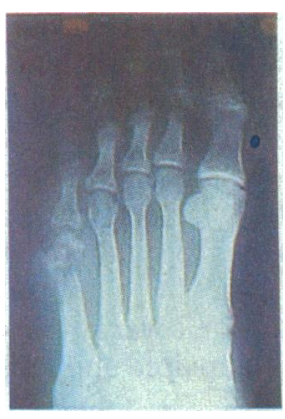

Radiograph showing bony destruction, particularly of the fifth metatarsal, and gas in the tissues as a result of ulceration.

\section{Other complications}

Painfut diabetic neuropathy - Though most diabetic peripheral neuropathy is painless, some patients have severe pain in the legs and feet; treatment of this is difficult and unsatisfactory. Optimal control of the diabetes is the first essential, together with simple analgesics and combinations of phenothiazines and antidepressants. Achievement of adequate sleep with night sedation is important. A new class of drugs, the aldose reductase inhibitors (which reduce accumulation of sorbitol in nerves affected by diabetes), is currently being evaluated in clinical trials.

Charcot's joints-Neurogenic arthropathy is a rare complication of diabetes that results in destruction of bones and joints leading to severe deformity of the foot. 


\section{The ischaemic foot}

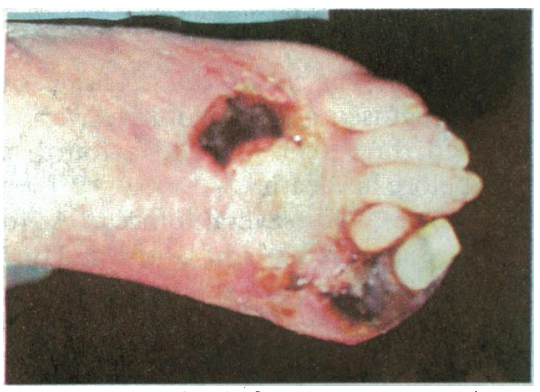

Ischaemic diabetic foot.

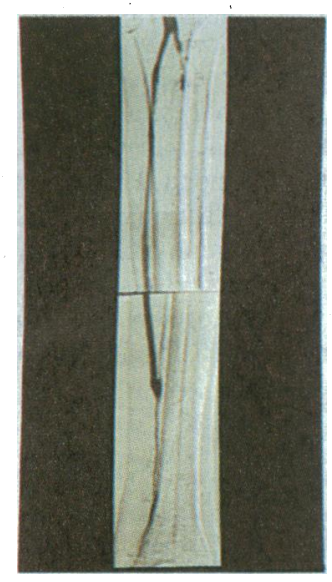

Angiogram of vein bypass graft from popliteal to posterior tibial artery. The popliteal pulse was present but the foot was ischaemic.

\section{Conclusion}

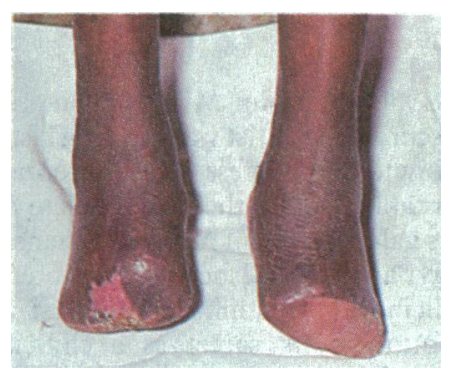

Bilateral leg revascularisation resulting in successful transmetatarsal amputations.
Ischaemia in a diabetic foot is caused by atherosclerosis, which is more common among diabetic patients than non-diabetic patients. The occlusive arterial disease occurs in younger patients and in a more severe form than in non-diabetic patients and it is more often bilateral. Furthermore, diabetic patients tend to have hypercoagulable blood. The symptoms are intermittent claudication and-later-rest pain, which may be followed by ulceration and gangrene. In contrast to the neuropathic foot, the ischaemic foot is cool with shiny red atrophic skin and absent peripheral pulses.

\section{Management}

Investigations-Doppler ultrasound pressure wave measurements of the ankle to arm systolic blood pressure ratio provide a simple non-invasive way of confirming the presence of peripheral vascular disease. Unfortunately the calcification that develops in these diabetic patients' vessels may make the vessels incompressible under the sphygmomanometer cuff and give spuriously normal readings. Arteriography is required if reconstructive surgery is to be considered. The presence of a palpable popliteal pulse is no longer a contraindication to arteriography, and if the femoral pulse is normal the radiologist should concentrate on the lower leg arteries, including those in the foot.

Medical management-Claudication is often mild and intermittent and may go on for years without progressing, and medical management has an important part to play. The patient must stop smoking, and control of the diabetes should be optimal. Infection should be treated early, and $\beta$ blockers should be avoided if possible. Suitable protective footwear should be worn and chiropody should be arranged. If the patient is obese, weight reduction should be encouraged until the weight is close to the ideal (which will also help improve control of the diabetes).

Surgical management - The previous pessimism about treating distal arterial occlusions by operation has been mitigated by improved techniques. Worthwhile results can now be expected if a vein graft can be anastomosed to a single patent vessel at the level of the ankle. A routine arteriogram may fail to opacify these vessels, but new arteriographic and Doppler techniques will show vessels that can be used for reconstruction. The "in situ" vein technique has helped, but arm veins may also be used and short bypasses from the popliteal artery to the ankle are also worth while.

The physician must be constantly alert to the development of peripheral neuropathy or vascular disease in diabetic patients. Examination of the feet should be part of every review in the diabetic clinic. Once a problem has arisen the patient should be instructed in the care of his or her feet. The patient should wear broad fitting shoes with low broad heels and soft uppers. There should be no pressure points. Printed instructions should be given to these patients.

Dr R S Elkeles, FRCP, is consultant physician, St Mary's Hospital, London, and Mr John H $\mathrm{N}$ Wolfe, FRCS, is consultant vascular surgeon, St Mary's Hospital, London.

The ABC of Vascular Diseases has been edited by Mr John H N Wolfe.

We acknowledge with thanks the assistance of the audiovisual department, St Mary's Hospital, London, in the preparation of the illustrations. We thank Upjohn for permission to reproduce the photograph depicting extensive destruction of tissue beneath small lesions and the radiograph. 